\title{
Molecular typing of Leishmania infantum isolates from a leishmaniasis outbreak in Madrid, Spain, 2009 to 2012
}

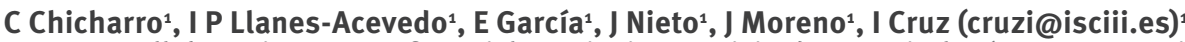

1. WHO Collaborating Centre for Leishmaniasis, Servicio de Parasitología, Centro Nacional de Microbiología, Instituto de Salud Carlos III, Madrid, Spain

Citation style for this article:

Chicharro C, Llanes-Acevedo IP, García E, Nieto J, Moreno J, Cruz I. Molecular typing of Leishmania infantum isolates from a leishmaniasis outbreak in Madrid, Spain, 2009 to 2012. Euro Surveill. 2013;18(30):pii=20545. Available online: http://www.eurosurveillance.org/ViewArticle.aspx?Articleld=20545

Leishmaniasis is endemic in south-west Europe. Recent data point to the spread and (re-)emergence of this disease in previously endemic and non-endemic European countries. A recent example is the urban community outbreak of cutaneous and visceral leishmaniasis in the south-west of Madrid autonomous community, Spain, which began on 1 July 2009. A total of 446 cases associated to this outbreak were reported up to 31 December 2012. We show molecular typing data for 73 Leishmania infantum isolates obtained from January 2008 to July 2012 from different areas of Madrid, including those affected by the outbreak. Seven different genotypes were identified by combining data from two targets: the ribosomal internal transcribed spacers (ITS) -1 and -2 and the haspb (k26) gene. The results contribute to a better understanding of the parasite population circulating in the region, and indicate that most of the outbreak-associated isolates $(22 / 31)$ were infected by parasites with the same combined genotype. Additional data from 82 L. infantum isolates typed as either MON-1 or MON-24 by isoenzyme analysis indicate that far from concluding that the outbreak was caused by a 'new' emerging genotype, further molecular typing-based surveillance studies are required to better understand the epidemiology of leishmaniasis in the region.

\section{Introduction}

Leishmania infantum is the causative agent of autochthonous cutaneous and visceral cases of leishmaniasis in Spain, and female sandflies of the species Phlebotomus perniciosus and $P$. ariasi are responsible of its transmission; this depends on a zoonotic cycle, in which dogs are considered the main reservoir hosts [1]. However, reports on Leishmania infection in other animals from Spain, such as wild carnivores, captive macropods, rabbits and hares, are increasing in number [2-5]. Although, their role in transmission to humans has yet to be elucidated, $L$. infantum transmission from naturally infected hares to $P$. perniciosus sandflies has been recently proven [5].

In Spain, leishmaniasis is considered a hypoendemic disease (0.41 cases per 100,000 inhabitants in 2012)
[1]. However, figures for both visceral (VL) and particularly cutaneous leishmaniasis (CL) are underestimates, due to the absence of a centralised surveillance system and because leishmaniasis is not a mandatorily notifiable disease in all autonomous communities of the country [1]. After the first description of a case of acquired immune deficiency syndrome (AIDS)associated leishmaniasis in 1985 [6], Spain faced a reemergence of leishmaniasis related to the spread of the human immunodeficiency virus (HIV). Of the 1,911 cases of coinfection reported from south-west Europe to the World Health Organization (WHO) between 1990 and 2001, Spain accounted for 1,099 of them [7]. Fortunately, the introduction of highly active antiretroviral therapy (HAART) therapy in the late 1990s contributed to a marked decrease of coinfection cases in south-west Europe: 299 cases were reported to WHO, 130 of which were from Spain, between 2001 and 2006 [8].

In spite of the worrying Leishmania/HIV coinfection phenomenon, leishmaniasis seems to have been under control in south-west Europe. Nevertheless, attention has been recently been drawn to the probable spread/ re-emergence of leishmaniasis in Europe, including discussion of the contributing factors $[9,10]$. At the same time, examples appeared, such as the northward spread of human and canine leishmaniasis in Italy (in 2003) [11,12] and canine leishmaniasis in Spain (in 2011) [13], and endemic transmission of L. infantum to dogs in Hungary (in 2007), which until then had been regarded as free of leishmaniasis $[14,15]$.

An urban community outbreak of $C L$ and $V L$ in the south-west of Madrid autonomous community (hereafter referred to as Madrid), Spain, provides a further example [16]. In Madrid, leishmaniasis surveillance has been carried out through a reporting system of mandatorily notifiable diseases since 1997, with regular records of 12 to 25 leishmaniasis cases per year [16]. However, in the last quarter of year 2010, a marked increase in the number of reported cases was noticed; subsequent investigation indicated that the outbreak had started on 1 July 2009 in the south-west of the 
Distribution by year, Health Area and pathology of the cases of leishmaniasis ${ }^{\text {, }}$ Madrid, Spain, 1 January $2008-31$ July 2012 $(\mathrm{n}=475)$

\begin{tabular}{|c|c|c|c|c|c|c|c|c|c|}
\hline \multirow{2}{*}{ Health Area } & \multicolumn{5}{|c|}{ Year } & \multirow{2}{*}{$\begin{array}{l}\text { Total per } \\
\text { Health Area }\end{array}$} & \multicolumn{3}{|c|}{ Clinical form of leishmaniasis } \\
\hline & 2008 & 2009 & 2010 & 2011 & 2012 & & $\mathrm{CL}$ & VL & Otherb \\
\hline$A_{1}$ & 2 & 4 & 0 & 0 & 0 & 6 & 0 & 6 & 0 \\
\hline$A_{2}$ & 2 & 0 & 3 & 5 & 0 & 10 & 3 & 7 & 0 \\
\hline A3 & 0 & 0 & 1 & 2 & 0 & 3 & 0 & 3 & 0 \\
\hline $\mathrm{A}_{4}$ & 4 & 10 & 3 & 5 & 4 & 26 & 6 & 18 & 2 \\
\hline$A_{5}$ & 20 & 8 & 13 & 11 & 6 & 58 & 13 & 45 & 0 \\
\hline A6 & 1 & 0 & 0 & 3 & 2 & 6 & 3 & 2 & 1 \\
\hline A7 & 1 & 3 & 1 & 2 & 2 & 9 & 1 & 3 & 5 \\
\hline A8 & 7 & 8 & 7 & 4 & 3 & 29 & 3 & 23 & 3 \\
\hline$A 9^{c}$ & 5 & 2 & 16 & 141 & 126 & 290 & 211 & 73 & 6 \\
\hline $\mathrm{A} 10^{c}$ & 3 & 1 & 2 & 9 & 10 & 25 & 10 & 14 & 1 \\
\hline A11 & 0 & 0 & 0 & 8 & 5 & 13 & 1 & 12 & 0 \\
\hline Total & 45 & 36 & 46 & 190 & 158 & 475 & 251 & 206 & 18 \\
\hline
\end{tabular}

CL: cutaneous leishmaniasis; VL: visceral leishmaniasis.

a Cases diagnosed during the period stated by polymerase chain reaction at the World Health Organization Collaborating Centre for Leishmaniasis in Madrid, Spain (Instituto de Salud Carlos III).

b 'Other' comprises mucosal leishmaniasis (ML), localised lymphadenopathy (LL) (A4: 2 ML in 2009; A6: 1 ML in 2008; A7: 2 LL in 2009-2011, and 3 ML in 2011-2012; A8: 1 LL in 2009 and 2 ML in 2009-2012; A9: 5 LL in 2010-2012, and 1 ML in 2011; A10: 1 LL in 2012).

c Health Areas 9 and 10 were affected by the outbreak that began on 1 July 2009.

region. The outbreak affected four geographically close municipalities, which share wide areas in urban green parks, with a population of half a million inhabitants [16]. As reported by Arce et al. [17], a total of 446 cases of leishmaniasis associated with this outbreak were reported up to 31 December 2012, with epidemic peaks in the winter of 2010 and 2011. Of the 446 cases, 160 (35.9\%) had visceral and $286(64.1 \%)$ cutaneous forms of the disease. The median age was 44 years (range: 2 months -95 years). Risk factors associated with immunosuppression appeared only in $15.2 \%$ of the cases.

The WHO Collaborating Centre for Leishmaniasis in Madrid (Instituto de Salud Carlos III), which acts as the reference laboratory for leishmaniasis in Spain, contributed to the diagnosis of the outbreak-associated cases through molecular and serological methods, as well as parasite isolation in culture. As part of these activities, we also performed molecular typing of an assembly of 73 isolates from the outbreak area and other regions of Madrid obtained from January 2008, the year before the outbreak started, to July 2012. The results of this investigation are here presented.

Concurrently, additional data from a second assembly of $83 \mathrm{~L}$. infantum human isolates, collected in Madrid between 1988 and 2005 and typed by multilocus enzyme electrophoresis (MLEE) as MON-1 and MON-24, zymodemes responsible of most of the $C L$ and $V L$ cases in Spain [18], are also presented.

\section{Methods}

First assembly: isolates from the outbreak area and other regions of Madrid, 2008-2012

In the WHO Collaborating Centre for Leishmaniasis, diagnosis of leishmaniasis is made on the basis of molecular and serological methods, as well as by isolation in Novy-MacNeal-Nicolle (NNN) culture. Additionally, Leishmania isolates are received from different hospitals to be kept in our cryobank.

From 1 January 2008 to 31 July 2012, we diagnosed a total of 475 cases of leishmaniasis in Madrid by polymerase chain reaction (PCR) [19]. Samples of patients came from the 11 Health Areas into which the region is divided. The mean number of cases for 2008 to 2010 was 42, while 190 were diagnosed in 2011 and 158 in the first six months of 2012; this large increase was related to the outbreak of leishmaniasis in Health Areas 9 and 10, in the south-west of the region. Of the 475 cases, 251 were CL, $206 \mathrm{VL}$ and 18 other forms of leishmaniasis (namely mucosal and localised lymphadenopathy). Details of the leishmaniasis cases diagnosed by PCR and their distribution by year and Health Area are shown in Table 1.

On the basis of the distribution of cases shown in Table 1 and on the availability of culture isolates successfully obtained from diagnostic samples at the WHO Collaborating Centre for Leishmaniasis or received 
TABLE 2A

Molecular typing of selected Leishmania infantum isolates from Madrid, Spain, obtained from 1 January $2008-31$ July 2012 $(n=73)$

\begin{tabular}{|c|c|c|c|c|c|c|c|c|}
\hline Isolate WHO code ${ }^{a}$ & $\begin{array}{l}\text { Year of } \\
\text { isolation }\end{array}$ & $\begin{array}{l}\text { Health } \\
\text { Areab of }^{b} \\
\text { isolate } \\
\text { origin }\end{array}$ & $\begin{array}{c}\text { Clinical } \\
\text { form of } \\
\text { leishmaniasis }\end{array}$ & HIV & $\begin{array}{l}\text { Age group } \\
\text { in years }\end{array}$ & ITS type & $\begin{array}{c}\text { haspb }(k 26) \\
\text { size in base } \\
\text { pairs }\end{array}$ & $\begin{array}{l}\text { Combined } \\
\text { genotype }\end{array}$ \\
\hline MHOM/ES/2008/LLM-1665 & 2008 & 1 & $\mathrm{VL}$ & NEG & $>18$ & LOMBARDI & 962 & $L-962$ \\
\hline MHOM/ES/2008/LLM-1695 & 2008 & 1 & $\mathrm{VL}$ & NEG & $>18$ & LOMBARDI & 836 & $L-836$ \\
\hline MHOM/ES/2008/LLM-1643 & 2008 & 5 & $\mathrm{VL}$ & POS & $>18$ & $\mathrm{~A}$ & 794 & A-794 \\
\hline MHOM/ES/2008/LLM-1657 & 2008 & 5 & $\mathrm{VL}$ & POS & $>18$ & LOMBARDI & 920 & $L-920$ \\
\hline MHOM/ES/2008/LLM-1676 & 2008 & 5 & $\mathrm{VL}$ & NEG & $>18$ & LOMBARDI & 920 & L-920 \\
\hline MHOM/ES/2008/LLM-1667 & 2008 & 5 & $\mathrm{VL}$ & POS & $>18$ & $\mathrm{~A}$ & 584 & A-584 \\
\hline MHOM/ES/2008/LLM-1644 & 2008 & 8 & $\mathrm{VL}$ & NEG & $>18$ & LOMBARDI & 920 & L-920 \\
\hline MHOM/ES/2008/LLM-1646 & 2008 & 8 & $\mathrm{CL}$ & POS & $>18$ & LOMBARDI & 920 & L-920 \\
\hline MHOM/ES/2008/LLM-1653 & 2008 & 8 & $\mathrm{VL}$ & POS & $>18$ & $\mathrm{~A}$ & 584 & A-584 \\
\hline MHOM/ES/2008/LLM-1681 & 2008 & 8 & $\mathrm{VL}$ & POS & $>18$ & A & 626 & A-626 \\
\hline MHOM/ES/2008/LLM-1648 & 2008 & 9 & $\mathrm{VL}$ & NEG & $>18$ & LOMBARDI & 920 & $\mathrm{~L}-920$ \\
\hline MHOM/ES/2009/LLM-1707 & 2009 & 1 & $\mathrm{VL}$ & POS & $>18$ & $\mathrm{~A}$ & 626 & A-626 \\
\hline MHOM/ES/2009/LLM-1725 & 2009 & 1 & $\mathrm{VL}$ & NEG & $>18$ & A & 626 & A-626 \\
\hline MHOM/ES/2009/LLM-1734 & 2009 & 1 & $\mathrm{VL}$ & NEG & $>18$ & $\mathrm{~A}$ & 626 & A-626 \\
\hline MHOM/ES/2009/LLM-1750 & 2009 & 1 & $\mathrm{VL}$ & NEG & $>18$ & LOMBARDI & 920 & L-920 \\
\hline MHOM/ES/2009/LLM-1729 & 2009 & 4 & $\mathrm{CL}$ & NEG & $>18$ & $\mathrm{~A}$ & 794 & A-794 \\
\hline MHOM/ES/2009/LLM-1756 & 2009 & 4 & VL & POS & $>18$ & A & 626 & A- 626 \\
\hline MHOM/ES/2009/LLM-1790 & 2009 & 5 & $\mathrm{CL}$ & POS & $>18$ & LOMBARDI & 920 & L-920 \\
\hline MHOM/ES/2009/LLM-1703 & 2009 & 8 & $\mathrm{VL}$ & POS & $>18$ & $\mathrm{~A}$ & 626 & A-626 \\
\hline MHOM/ES/2009/LLM-1712 & 2009 & 8 & $\mathrm{VL}$ & NEG & $>18$ & $\mathrm{~A}$ & 626 & A-626 \\
\hline MHOM/ES/2009/LLM-1714 & 2009 & 8 & VL & NEG & $>18$ & LOMBARDI & 920 & L-920 \\
\hline MHOM/ES/2009/LLM-1896 & 2010 & 1 & $\mathrm{VL}$ & NEG & $>18$ & $\mathrm{~A}$ & 626 & A-626 \\
\hline MHOM/ES/2010/LLM-1920 & 2010 & 2 & $\mathrm{VL}$ & NEG & $<5$ & LOMBARDI & 962 & $\mathrm{~L}-962$ \\
\hline MHOM/ES/2010/LLM-1873 & 2010 & 4 & VL & NEG & $>18$ & A & 626 & A-626 \\
\hline MHOM/ES/2010/LLM-1858 & 2010 & 5 & $\mathrm{VL}$ & NEG & $>18$ & $\mathrm{~A}$ & 626 & A- 626 \\
\hline MHOM/ES/2010/LLM-1859 & 2010 & 8 & $\mathrm{VL}$ & NEG & $<5$ & LOMBARDI & $962 / 920$ & $L-962 / 920$ \\
\hline MHOM/ES/2010/LLM-1888 & 2010 & 8 & $\mathrm{VL}$ & NEG & $<5$ & LOMBARDI & 962 & $\mathrm{~L}-962$ \\
\hline MHOM/ES/2010/LLM-1854 & 2010 & 9 & $\mathrm{VL}$ & NEG & $>18$ & LOMBARDI & 920 & $L-920$ \\
\hline MHOM/ES/2010/LLM-1918 & 2010 & 9 & $\mathrm{LL}$ & NEG & $>18$ & LOMBARDI & 962 & $L-962$ \\
\hline MHOM/ES/2010/LLM-1886 & 2010 & 9 & $\mathrm{VL}$ & NEG & $>18$ & LOMBARDI & 920 & $L-920$ \\
\hline MHOM/ES/2010/LLM-1899 & 2010 & 9 & VL & POS & $>18$ & LOMBARDI & 920 & L-920 \\
\hline MHOM/ES/2011/LLM-2027 & 2011 & 1 & $\mathrm{VL}$ & NEG & $>18$ & $\mathrm{~A}$ & 626 & A-626 \\
\hline MHOM/ES/2011/LLM-2032 & 2011 & 1 & $\mathrm{VL}$ & NEG & $>18$ & LOMBARDI & $962 / 920$ & $L-962 / 920$ \\
\hline MHOM/ES/2011/LLM-2005 & 2011 & 3 & $\mathrm{VL}$ & NEG & $<5$ & LOMBARDI & 920 & L-920 \\
\hline MHOM/ES/2011/LLM-2051 & 2011 & 4 & $\mathrm{VL}$ & NEG & $>18$ & A & 626 & A- 626 \\
\hline MHOM/ES/2011/LLM-1948 & 2011 & 5 & $\mathrm{CL}$ & NEG & $>18$ & A & 626 & A-626 \\
\hline MHOM/ES/2011/LLM-2033 & 2011 & 5 & $\mathrm{VL}$ & NEG & $<5$ & A & 626 & A-626 \\
\hline MHOM/ES/2011/LLM-2047 & 2011 & 5 & $\mathrm{VL}$ & POS & $>18$ & $\mathrm{~A}$ & 626 & A- 626 \\
\hline MHOM/ES/2011/LLM-1946 & 2011 & 7 & $\mathrm{LL}$ & NEG & $>18$ & LOMBARDI & 920 & L-920 \\
\hline MHOM/ES/2011/LLM-2018 & 2011 & 8 & VL & NEG & $>18$ & LOMBARDI & 920 & L-920 \\
\hline MHOM/ES/2011/LLM-2037 & 2011 & 9 & $\mathrm{VL}$ & NEG & $>18$ & LOMBARDI & 920 & L-920 \\
\hline MHOM/ES/2011/LLM-1988 & 2011 & 9 & VL & NEG & $>18$ & LOMBARDI & 962 & $L-962$ \\
\hline MHOM/ES/2011/LLM-1964 & 2011 & 9 & $\mathrm{VL}$ & NEG & $>18$ & LOMBARDI & 836 & L-836 \\
\hline
\end{tabular}

CL: cutaneous leishmaniasis; HIV: human immunodeficiency virus; haspb: hydrophilic acylated surface protein B gene; ITS: ribosomal internal transcribed spacers; LL: localised lymphadenopathy; ML: mucosal leishmaniasis; NEG: negative; POS: positive; VL: visceral leishmaniasis; WHO: World Health Organization.

a Isolates ordered by year of isolation and Health Area.

b Health Areas 9 and 10 were affected by the outbreak that began on 1 July 2009.

Combined genotypes are derived from combining the results of ITS sequence type and haspb (k26) polymerase chain reaction product size. 


\section{TABLE 2B}

Molecular typing of selected Leishmania infantum isolates from Madrid, Spain, obtained from 1 January 2008-31 July 2012 $(\mathrm{n}=73)$

\begin{tabular}{|c|c|c|c|c|c|c|c|c|}
\hline Isolate WHO code ${ }^{\mathrm{a}}$ & $\begin{array}{l}\text { Year of } \\
\text { isolation }\end{array}$ & $\begin{array}{l}\text { Health } \\
\text { Area }^{b} \text { of } \\
\text { isolate } \\
\text { origin }\end{array}$ & $\begin{array}{c}\text { Clinical } \\
\text { form of } \\
\text { leishmaniasis }\end{array}$ & HIV & $\begin{array}{l}\text { Age group } \\
\text { in years }\end{array}$ & ITS type & $\begin{array}{l}\text { haspb (k26) } \\
\text { size in base } \\
\text { pairs }\end{array}$ & $\begin{array}{l}\text { Combined } \\
\text { genotype }^{c}\end{array}$ \\
\hline MHOM/ES/2011/LLM-1929 & 2011 & 9 & $\mathrm{VL}$ & NEG & $>18$ & LOMBARDI & 920 & L-920 \\
\hline MHOM/ES/2011/LLM-1956 & 2011 & 9 & $\mathrm{VL}$ & NEG & $>18$ & LOMBARDI & 920 & L-920 \\
\hline MHOM/ES/2011/LLM-1974 & 2011 & 9 & $\mathrm{CL}$ & NEG & $>18$ & LOMBARDI & 920 & L-920 \\
\hline MHOM/ES/2011/LLM-1982 & 2011 & 9 & LL & NEG & $>18$ & LOMBARDI & 920 & L-920 \\
\hline MHOM/ES/2011/LLM-1983 & 2011 & 9 & $\mathrm{CL}$ & NEG & $>18$ & LOMBARDI & 920 & L-920 \\
\hline MHOM/ES/2011/LLM-1984 & 2011 & 9 & VL & POS & $>18$ & LOMBARDI & $962 / 920$ & $L-962 / 920$ \\
\hline MHOM/ES/2011/LLM-1998 & 2011 & 9 & $\mathrm{ML}$ & NEG & $>18$ & LOMBARDI & $962 / 920$ & L-962/920 \\
\hline MHOM/ES/2011/LLM-2001 & 2011 & 9 & $\mathrm{CL}$ & NEG & $>18$ & LOMBARDI & 920 & L-920 \\
\hline MHOM/ES/2011/LLM-2039 & 2011 & 9 & $\mathrm{VL}$ & NEG & $>18$ & LOMBARDI & $962 / 920$ & $L-962 / 920$ \\
\hline MHOM/ES/2011/LLM-2046 & 2011 & 9 & $\mathrm{CL}$ & NEG & 218 & LOMBARDI & 920 & L-920 \\
\hline MHOM/ES/2011/LLM-2048 & 2011 & 9 & $\mathrm{CL}$ & NEG & $>18$ & LOMBARDI & 920 & L-920 \\
\hline MHOM/ES/2011/LLM-1937 & 2011 & 10 & $\mathrm{VL}$ & NEG & $>18$ & LOMBARDI & $962 / 920$ & $L-962 / 920$ \\
\hline MHOM/ES/2011/LLM-1954 & 2011 & 10 & $\mathrm{VL}$ & NEG & $>18$ & LOMBARDI & 962 & L-962 \\
\hline MHOM/ES/2011/LLM-2025 & 2011 & 11 & $\mathrm{VL}$ & NEG & $5-18$ & LOMBARDI & $962 / 920$ & $L-962 / 920$ \\
\hline MHOM/ES/2012/LLM-2096 & 2012 & 1 & $\mathrm{VL}$ & NEG & $>18$ & LOMBARDI & 920 & L-920 \\
\hline MHOM/ES/2012/LLM-2134 & 2012 & 1 & $\mathrm{VL}$ & POS & $>18$ & LOMBARDI & 920 & L-920 \\
\hline MHOM/ES/2012/LLM-2098 & 2012 & 5 & $\mathrm{VL}$ & NEG & $<5$ & $\mathrm{~A}$ & 626 & A- 626 \\
\hline MHOM/ES/2012/LLM-2118 & 2012 & 6 & $\mathrm{CL}$ & NEG & $>18$ & $\mathrm{~A}$ & 626 & A- 626 \\
\hline MHOM/ES/2012/LLM-2109 & 2012 & 6 & $\mathrm{VL}$ & NEG & $>18$ & LOMBARDI & 962 & L-962 \\
\hline MHOM/ES/2012/LLM-2139 & 2012 & 9 & $\mathrm{VL}$ & NEG & $>18$ & LOMBARDI & 920 & L-920 \\
\hline MHOM/ES/2012/LLM-2059 & 2012 & 9 & $\mathrm{CL}$ & NEG & $>18$ & LOMBARDI & 920 & L-920 \\
\hline MHOM/ES/2012/LLM-2063 & 2012 & 9 & $\mathrm{VL}$ & NEG & $>18$ & LOMBARDI & 920 & L-920 \\
\hline MHOM/ES/2012/LLM-2064 & 2012 & 9 & $\mathrm{CL}$ & NEG & $>18$ & LOMBARDI & 920 & L-920 \\
\hline MHOM/ES/2012/LLM-2072 & 2012 & 9 & $\mathrm{CL}$ & NEG & $>18$ & LOMBARDI & 920 & L-920 \\
\hline MHOM/ES/2012/LLM-2074 & 2012 & 9 & $\mathrm{CL}$ & NEG & $>18$ & LOMBARDI & 920 & L-920 \\
\hline MHOM/ES/2012/LLM-2076 & 2012 & 9 & $\mathrm{CL}$ & NEG & $>18$ & LOMBARDI & 920 & L-920 \\
\hline MHOM/ES/2012/LLM-2077 & 2012 & 9 & $\mathrm{CL}$ & NEG & $>18$ & LOMBARDI & 920 & L-920 \\
\hline MHOM/ES/2012/LLM-2079 & 2012 & 9 & $\mathrm{VL}$ & POS & $>18$ & LOMBARDI & 920 & L-920 \\
\hline MHOM/ES/2012/LLM-2097 & 2012 & 9 & $\mathrm{VL}$ & NEG & $>18$ & LOMBARDI & $962 / 920$ & L-962/920 \\
\hline MHOM/ES/2012/LLM-2113 & 2012 & 9 & $\mathrm{VL}$ & NEG & $>18$ & LOMBARDI & 920 & L-920 \\
\hline
\end{tabular}

CL: cutaneous leishmaniasis; HIV: human immunodeficiency virus; haspb: hydrophilic acylated surface protein B gene; ITS: ribosomal internal transcribed spacers; LL: localised lymphadenopathy; ML: mucosal leishmaniasis; NEG: negative; POS: positive; VL: visceral leishmaniasis; WHO: World Health Organization.

a Isolates ordered by year of isolation and Health Area.

b Health Areas 9 and 10 were affected by the outbreak that began on 1 July 2009.

c Combined genotypes are derived from combining the results of ITS sequence type and haspb (k26) polymerase chain reaction product size. 
Distribution and combined genotype of analysed Leishmania infantum isolates from Madrid, Spain,

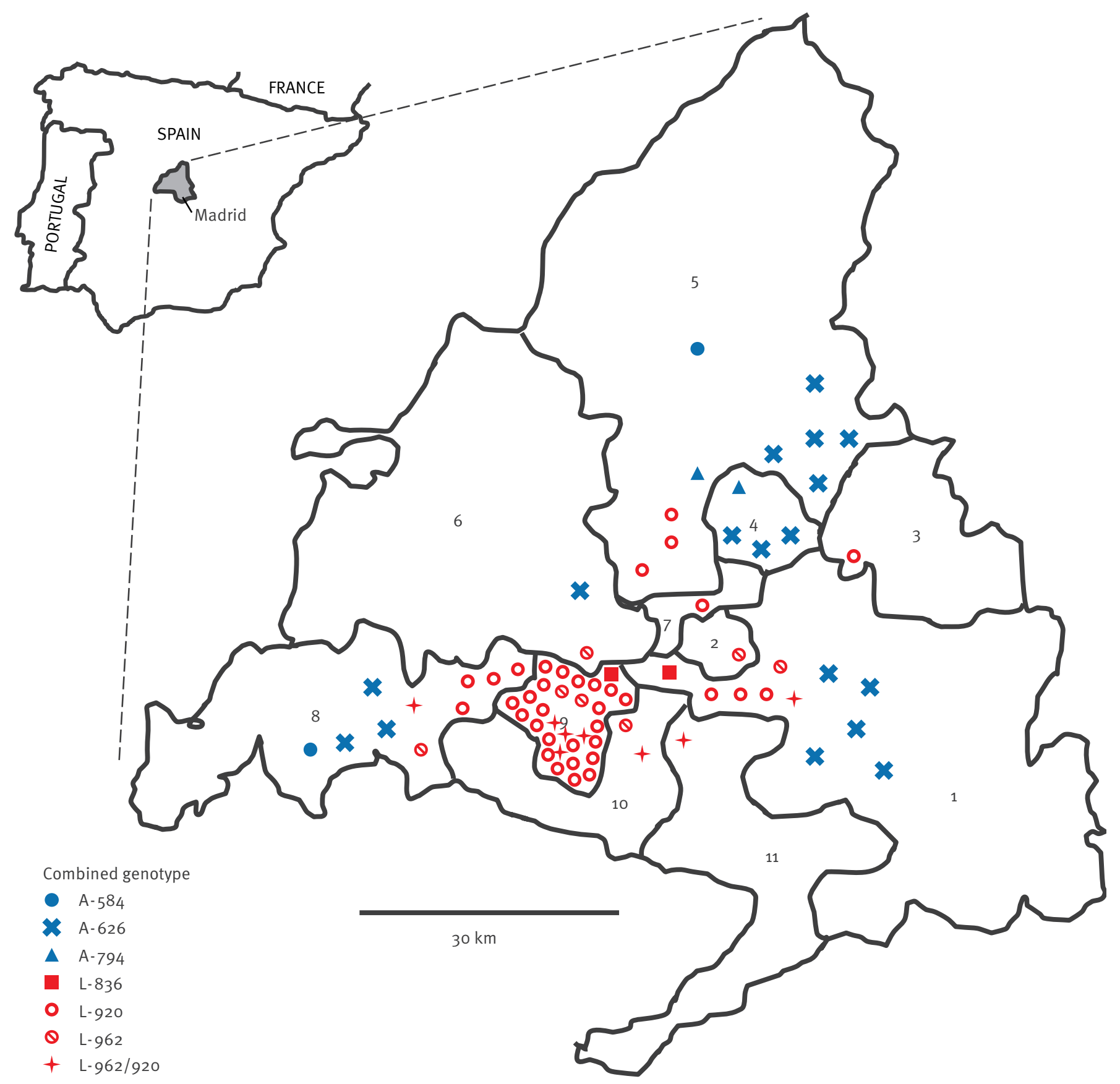

The numbers 1-11 represent the 11 Health Areas in Madrid. The combined genotypes of the isolates are shown. 
from referral hospitals, we prepared an assembly of Leishmania isolates from the cases diagnosed by the WHO Collaborating Centre during 1 January 2008 to 31 July 2012. The assembly was not solely focused on the Health Areas affected by the outbreak (Health Areas 9 and 10): in order to have a fuller picture of the context in which the outbreak occurred, isolates from all 11 Health Areas in Madrid were included. For the same reason, we also decided to include isolates obtained in 2008 (the year before the outbreak started). A total of $73 \mathrm{~L}$. infantum isolates were included in our analysis: 16 from CL patients, 53 from VL patients, 3 from patients affected by localised lymphadenopathy and 1 from a patient with mucosal leishmaniasis. A total of 15 isolates were obtained from patients with concomitant human immunodeficiency virus (HIV) infection. Further details of the isolates included in the assembly for molecular typing concerning year of isolation, Health Area of origin, clinical form of leishmaniasis, HIV status, age and sex are shown in Table 2 and Figure 1.

\section{Second assembly: L. infantum}

MON-1 and MON-24 isolates, 1988-2005

The intraspecies variability of $L$. infantum has long been studied by MLEE. This approach, and the subsequent identification of different zymodemes, constituted an extremely useful taxonomic tool, which has contributed much to understand the epidemiology of leishmaniasis $[20,21]$. Although MLEE has been used for Leishmania typing during the past 25 years, some drawbacks have been attributed to this methodology when compared with molecular methods [22], which are being increasingly used for epidemiological studies of visceral and cutaneous leishmaniases and are widely available in various laboratories.

As L. infantum MON-1 and MON-24 zymodemes are known to be responsible for most $C L$ and $V L$ cases in Spain [18], we also included in this study 83 L. infantum isolates from Madrid that had been typed by MLEE as MON-1 $(n=55)$ and MON-24 $(n=28)$ at the WHO Collaborating Centre for Leishmaniasis, to have a reference at the MLEE level in our analysis. Details of these isolates are given in Table 3 and Figure 2.

\section{DNA extraction}

DNA was extracted from culture pellets cryopreserved at the WHO Collaborating Centre for Leishmaniasis using the QIAamp DNA mini kit (QIAGEN). The DNA was eluted in PCR-grade water and adjusted to a final concentration of $10 \mathrm{ng} / \mu \mathrm{l}$ with NanoDrop ND-1000 spectrophotometer (NanoDrop Technologies). For further PCR reactions, $5 \mu \mathrm{l}$ of each DNA sample were used.

\section{Molecular typing}

Two different targets of the Leishmania genome were selected for molecular typing analyses. ITS1 and ITS2 were amplified following the protocol described by Kuhls et al. [23] and the haspb (k26) gene (hydrophilic acylated surface protein B) was amplified as described by Haralambous et al. [24]. For species identification of isolates from the first assembly, the heat shock protein 70 ( $h s p 70$ ) gene was amplified as described by Fraga et al. [25]; species identification was not performed on the second assembly of isolates because they had been previously typed as L. infantum MON-1 or MON24 by MLEE. PCR products were run on $2 \%$ agarose gels stained with ethidium bromide and visualised under ultraviolet light.

Direct sequencing of the ITS1, ITS2 and hsp7o PCR products was performed with the corresponding forward and reverse primers; internal primers for sequencing were also used for hsp7o, as described elsewhere [25]. Before DNA sequencing, the PCR products were excised from agarose gels and purified using the QIAquick Gel Extraction Kit (QIAGEN). The Big-Dye Terminator Cycle Sequencing Ready Reaction Kit V3.1 and the automated ABI PRISM 377 DNA sequencer (Applied Biosystems) were used. Sequences obtained were analysed and edited using the software BioEdit Sequence Alignment Editor, version 7.0.9.0 [26]. ITS1 and ITS2 sequences were compared with sequences of the ITS types described by Kuhls et al. [23], these sequences were aligned with BioEdit Sequence Alignment Editor using ClustalW multiple alignment algorithm and were manually adjusted.

For accurate estimation of the $k 26$ PCR product size, the bands were excised from agarose gels, purified using the QIAquick Gel Extraction Kit (QIAGEN), and analysed by capillary electrophoresis using the Agilent 2100 Bioanalyzer and the Agilent DNA 1000 kit (Agilent Technologies).

ITS genotypes were assigned to each isolate according to the sequence polymorphism of the 12 microsatellite regions in ITS1 (four sites) and ITS2 (eight sites). k26 genotypes were assigned according to the size of the PCR product, and adjusted considering the gene size variability is due to the number of 42 nucleotide repeated motifs [24,27-29]. A combined genotype, derived from combining the results of ITS sequence type and k26 PCR product size, was then assigned to each isolate (first assembly only).

For the second assembly, we present data on ITS genotypes only; information on the $k 26$ gene analysis will be published separately.

\section{Phylogenetic analysis}

Phylogenetic analysis based on the nucleotide sequences of the Leishmania ITS1 and ITS2 was performed based on maximum parsimony using PHYLIP (PHYLogeny Inference Package), version 3.69 [30]. To test the robustness of the internal branches generated, we performed bootstrap analysis using 10,000 replications. We included DNA sequences retrieved from GenBank (AJ634341, AJooo288, AJoo0294, AJ634356, AJ634367, AJ634373, AJoo0297, AJ634376) that are representative for each of the ITS types $A$ to $H$, respectively, described by Kuhls et al. [23]. According to these 
TABLE 3A

Selected Leishmania infantum MON-1 and MON-24 isolates from Madrid, Spain, 1988-2005 (n=83)

\begin{tabular}{|c|c|c|c|c|c|c|c|}
\hline Isolate WHO code ${ }^{a}$ & $\begin{array}{l}\text { Year of } \\
\text { isolation }\end{array}$ & $\begin{array}{l}\text { Health Area of } \\
\text { isolate origin }\end{array}$ & $\begin{array}{c}\text { Clinical } \\
\text { form of } \\
\text { leishmaniasis }\end{array}$ & HIV & $\begin{array}{l}\text { Age group } \\
\text { in years }\end{array}$ & Zymodeme & ITS type \\
\hline MHOM/ES/1988/LLM-180 & 1988 & 1 & $\mathrm{VL}$ & NEG & $>18$ & MON-1 & A \\
\hline MHOM/ES/1990/LLM-195 & 1990 & 1 & $\mathrm{VL}$ & Unknown & Unknown & MON-1 & A \\
\hline MHOM/ES/1991/LLM-326 & 1991 & 4 & VL & POS & Unknown & MON-1 & A \\
\hline MHOM/ES/1991/LLM-328 & 1991 & 4 & $\mathrm{VL}$ & NEG & Unknown & MON-1 & A \\
\hline MHOM/ES/1992/LLM-339 & 1992 & 1 & $\mathrm{VL}$ & Unknown & Unknown & MON-1 & $\mathrm{A}$ \\
\hline MHOM/ES/1992/LLM-335 & 1992 & 1 & $\mathrm{VL}$ & POS & Unknown & MON-1 & LOMBARDI \\
\hline MHOM/ES/1992/LLM-315 & 1992 & 4 & $\mathrm{VL}$ & Unknown & Unknown & MON-1 & LOMBARDI \\
\hline MHOM/ES/1992/LLM-323 & 1992 & 5 & $\mathrm{VL}$ & POS & $>18$ & MON-1 & $\mathrm{A}$ \\
\hline MHOM/ES/1992/LLM-306 & 1992 & 5 & $\mathrm{VL}$ & POS & Unknown & MON-1 & A \\
\hline MHOM/ES/1993/LLM-404 & 1993 & 5 & $\mathrm{VL}$ & POS & Unknown & MON-1 & A \\
\hline MHOM/ES/1994/LLM-410 & 1994 & 5 & VL & POS & $>18$ & MON-1 & A \\
\hline MHOM/ES/1995/LLM-442 & 1995 & 2 & VL & POS & $>18$ & MON-1 & A \\
\hline MHOM/ES/1995/LLM-468 & 1995 & 3 & $\mathrm{VL}$ & Unknown & Unknown & MON-1 & A \\
\hline MHOM/ES/1995/LLM-450 & 1995 & 4 & $\mathrm{VL}$ & POS & Unknown & MON-1 & A \\
\hline MHOM/ES/1995/LLM-464 & 1995 & 5 & $\mathrm{VL}$ & POS & $>18$ & MON-1 & A \\
\hline MHOM/ES/1995/LLM-470 & 1995 & 5 & $\mathrm{VL}$ & POS & $>18$ & MON-1 & A \\
\hline MHOM/ES/1995/LLM-482 & 1995 & 5 & $\mathrm{VL}$ & NEG & $>18$ & MON-1 & A \\
\hline MHOM/ES/1996/LLM-554 & 1996 & 4 & $\mathrm{VL}$ & POS & Unknown & MON-1 & A \\
\hline MHOM/ES/1996/LLM-549 & 1996 & 5 & $\mathrm{VL}$ & POS & $>18$ & MON-1 & A \\
\hline MHOM/ES/1996/LLM-556 & 1996 & 5 & VL & POS & $>18$ & MON-1 & A \\
\hline MHOM/ES/1996/LLM-548 & 1996 & 5 & $\mathrm{VL}$ & POS & Unknown & MON-1 & A \\
\hline MHOM/ES/1997/LLM-607 & 1997 & 4 & $\mathrm{CL}$ & POS & $>18$ & MON-1 & $\mathrm{A}$ \\
\hline MHOM/ES/1997/LLM-465 & 1997 & 5 & $\mathrm{VL}$ & POS & $>18$ & MON-1 & LOMBARDI \\
\hline MHOM/ES/1997/LLM-616 & 1997 & 5 & VL & POS & $>18$ & MON-1 & $A$ \\
\hline MHOM/ES/1997/LLM-623 & 1997 & 5 & VL & POS & Unknown & MON-1 & A \\
\hline MHOM/ES/1997/LLM-674 & 1997 & 5 & $\mathrm{VL}$ & POS & $>18$ & MON-1 & A \\
\hline MHOM/ES/1997/LLM-666 & 1997 & 5 & $\mathrm{CL}$ & POS & Unknown & MON-1 & A \\
\hline MHOM/ES/1997/LLM-665 & 1997 & 9 & $\mathrm{VL}$ & POS & $5-18$ & MON-1 & $\mathrm{A}$ \\
\hline MHOM/ES/1997/LLM-690 & 1997 & 11 & $\mathrm{VL}$ & POS & $>18$ & MON-1 & LOMBARDI \\
\hline MHOM/ES/1998/LLM-739 & 1998 & 3 & $\mathrm{VL}$ & POS & $>18$ & MON-1 & $\mathrm{A}$ \\
\hline MHOM/ES/1999/LLM-755 & 1998 & 5 & $\mathrm{VL}$ & POS & Unknown & MON-1 & $\mathrm{A}$ \\
\hline MHOM/ES/1998/LLM-789 & 1998 & 8 & $\mathrm{VL}$ & NEG & $>18$ & MON-1 & $\mathrm{A}$ \\
\hline MHOM/ES/1999/LLM-896 & 1999 & 5 & $\mathrm{VL}$ & POS & Unknown & MON-1 & LOMBARDI \\
\hline MHOM/ES/1999/LLM-883 & 1999 & 8 & $\mathrm{CL}$ & POS & $>18$ & MON-1 & $\mathrm{A}$ \\
\hline MHOM/ES/1999/LLM-826 & 1999 & 11 & $\mathrm{VL}$ & POS & $>18$ & MON-1 & $\mathrm{A}$ \\
\hline MHOM/ES/2000/LLM-936 & 2000 & 4 & $\mathrm{VL}$ & Unknown & Unknown & MON-1 & LOMBARDI \\
\hline MHOM/ES/2001/LLM-983 & 2001 & 5 & $\mathrm{VL}$ & POS & $>18$ & MON-1 & $A$ \\
\hline MHOM/ES/2001/LLM-980 & 2001 & 8 & VL & NEG & $>18$ & MON-1 & LOMBARDI \\
\hline MHOM/ES/2001/LLM-984 & 2001 & 8 & $\mathrm{VL}$ & NEG & $>18$ & MON-1 & LOMBARDI \\
\hline MHOM/ES/2002/LLM-1220 & 2002 & 1 & $\mathrm{VL}$ & POS & Unknown & MON-1 & $\mathrm{A}$ \\
\hline MHOM/ES/2002/LLM-1181 & 2002 & 1 & $\mathrm{VL}$ & POS & Unknown & MON-1 & $\mathrm{A}$ \\
\hline MHOM/ES/2002/LLM-1166 & 2002 & 5 & $\mathrm{VL}$ & POS & $>18$ & MON-1 & LOMBARDI \\
\hline MHOM/ES/2003/LLM-1262 & 2003 & 1 & $\mathrm{VL}$ & POS & Unknown & MON-1 & $\mathrm{A}$ \\
\hline MHOM/ES/2003/LLM-1304 & 2003 & 1 & $\mathrm{VL}$ & NEG & $>18$ & MON-1 & LOMBARDI \\
\hline
\end{tabular}

CL: cutaneous leishmaniasis; HIV: human immunodeficiency virus; ITS: ribosomal internal transcribed spacers; NEG: negative; POS: positive; VL: visceral leishmaniasis; WHO: World Health Organization.

${ }^{\text {a }}$ Isolates ordered by year of isolation, Health Area and zymodeme. 
TABLE 3B

Selected Leishmania infantum MON-1 and MON-24 isolates from Madrid, Spain, 1988-2005 (n=83)

\begin{tabular}{|c|c|c|c|c|c|c|c|}
\hline Isolate WHO code ${ }^{\mathrm{a}}$ & $\begin{array}{l}\text { Year of } \\
\text { isolation }\end{array}$ & $\begin{array}{l}\text { Health Area of } \\
\text { isolate origin }\end{array}$ & $\begin{array}{c}\text { Clinical } \\
\text { form of } \\
\text { leishmaniasis }\end{array}$ & HIV & $\begin{array}{l}\text { Age group } \\
\text { in years }\end{array}$ & Zymodeme & ITS type \\
\hline MHOM/ES/2003/LLM-1327 & 2003 & 5 & $\mathrm{VL}$ & NEG & $<5$ & MON-1 & $\mathrm{A}$ \\
\hline MHOM/ES/2003/LLM-1258 & 2003 & 8 & $\mathrm{VL}$ & NEG & $>18$ & MON-1 & LOMBARDI \\
\hline MHOM/ES/2004/LLM-1372 & 2004 & 1 & VL & POS & $>18$ & MON-1 & A \\
\hline MHOM/ES/2004/LLM-1377 & 2004 & 1 & VL & POS & $>18$ & MON-1 & A \\
\hline MHOM/ES/2004/LLM-1337 & 2004 & 5 & $\mathrm{VL}$ & POS & Unknown & MON-1 & A \\
\hline MHOM/ES/2004/LLM-1461 & 2004 & 5 & $\mathrm{VL}$ & POS & Unknown & MON-1 & A \\
\hline MHOM/ES/2004/LLM-1405 & 2004 & 5 & $\mathrm{VL}$ & POS & Unknown & MON-1 & A \\
\hline MHOM/ES/2004/LLM-1347 & 2004 & 10 & $\mathrm{VL}$ & POS & Unknown & MON-1 & $\mathrm{A}$ \\
\hline MHOM/ES/2005/LLM-1524 & 2005 & 1 & $\mathrm{VL}$ & Unknown & $>18$ & MON-1 & $A$ \\
\hline MHOM/ES/2005/LLM-1523 & 2005 & 1 & $\mathrm{VL}$ & Unknown & $>18$ & MON-1 & A \\
\hline MHOM/ES/2005/LLM-1492 & 2005 & 8 & $\mathrm{VL}$ & POS & Unknown & MON-1 & $\mathrm{A}$ \\
\hline MHOM/ES/1995/LLM-443 & 1995 & 4 & VL & POS & Unknown & MON-24 & LOMBARDI \\
\hline MHOM/ES/1995/LLM-441 & 1995 & 5 & VL & POS & Unknown & MON-24 & LOMBARDI \\
\hline MHOM/ES/1995/LLM-485 & 1995 & 5 & $\mathrm{VL}$ & POS & $>18$ & MON-24 & LOMBARDI \\
\hline MHOM/ES/1995/LLM-465 & 1995 & 5 & $\mathrm{VL}$ & POS & $>18$ & MON-24 & LOMBARDI \\
\hline MHOM/ES/1995/LLM-456 & 1995 & 8 & $\mathrm{VL}$ & NEG & 218 & MON-24 & LOMBARDI \\
\hline MHOM/ES/1996/LLM-587 & 1996 & 2 & $\mathrm{CL}$ & POS & 218 & MON-24 & LOMBARDI \\
\hline MHOM/ES/1996/LLM-576 & 1996 & 5 & $\mathrm{VL}$ & POS & $>18$ & MON-24 & LOMBARDI \\
\hline MHOM/ES/1996/LLM-598 & 1996 & 5 & VL & POS & $>18$ & MON-24 & LOMBARDI \\
\hline MHOM/ES/1996/LLM-569 & 1996 & 11 & $\mathrm{VL}$ & POS & Unknown & MON-24 & LOMBARDI \\
\hline MHOM/ES/1997/LLM-713 & 1997 & 2 & $\mathrm{CL}$ & POS & $>18$ & MON-24 & LOMBARDI \\
\hline MHOM/ES/1997/LLM-711 & 1997 & 11 & VL & POS & $>18$ & MON-24 & LOMBARDI \\
\hline MHOM/ES/1998/LLM-779 & 1998 & 8 & $\mathrm{VL}$ & POS & $>18$ & MON-24 & LOMBARDI \\
\hline MHOM/ES/1998/LLM-730 & 1998 & 11 & $\mathrm{VL}$ & POS & $>18$ & MON-24 & LOMBARDI \\
\hline MHOM/ES/1999/LLM-845 & 1999 & 8 & $\mathrm{VL}$ & POS & Unknown & MON-24 & LOMBARDI \\
\hline MHOM/ES/2000/LLM-957 & 2000 & 5 & VL & Unknown & Unknown & MON-24 & LOMBARDI \\
\hline MHOM/ES/2001/LLM-1078 & 2001 & 1 & $\mathrm{VL}$ & POS & Unknown & MON-24 & LOMBARDI \\
\hline MHOM/ES/2001/LLM-1065 & 2001 & 5 & $\mathrm{VL}$ & POS & $>18$ & MON-24 & LOMBARDI \\
\hline MHOM/ES/2001/LLM-1027 & 2001 & 8 & $\mathrm{VL}$ & POS & Unknown & MON-24 & LOMBARDI \\
\hline MHOM/ES/2001/LLM-1032 & 2001 & 8 & $\mathrm{VL}$ & POS & Unknown & MON-24 & LOMBARDI \\
\hline MHOM/ES/2001/LLM-982 & 2001 & 1 & $\mathrm{VL}$ & POS & $>18$ & MON-24 & LOMBARDI \\
\hline MHOM/ES/2002/LLM-1177 & 2002 & 8 & $\mathrm{CL}$ & NEG & Unknown & MON-24 & LOMBARDI \\
\hline MHOM/ES/2003/LLM-1305 & 2003 & 5 & VL & POS & $>18$ & MON-24 & LOMBARDI \\
\hline MHOM/ES/2004/LLM-1346 & 2004 & 3 & VL & POS & $>18$ & MON-24 & LOMBARDI \\
\hline MHOM/ES/2004/LLM-1367 & 2004 & 8 & VL & POS & Unknown & MON-24 & LOMBARDI \\
\hline MHOM/ES/2005/LLM-1475 & 2005 & 5 & $\mathrm{CL}$ & NEG & 45 & MON-24 & LOMBARDI \\
\hline MHOM/ES/2005/LLM-1526 & 2005 & 1 & $\mathrm{VL}$ & Unknown & Unknown & MON-24 & LOMBARDI \\
\hline MHOM/ES/2005/LLM-1478 & 2005 & 8 & VL & NEG & $>18$ & MON-24 & LOMBARDI \\
\hline MHOM/ES/2005/LLM-1525 & 2005 & 10 & $\mathrm{VL}$ & NEG & Unknown & MON-24 & LOMBARDI \\
\hline
\end{tabular}

CL: cutaneous leishmaniasis; HIV: human immunodeficiency virus; ITS: ribosomal internal transcribed spacers; NEG: negative; POS: positive; VL: visceral leishmaniasis; WHO: World Health Organization.

${ }^{a}$ Isolates ordered by year of isolation, Health Area and zymodeme. 


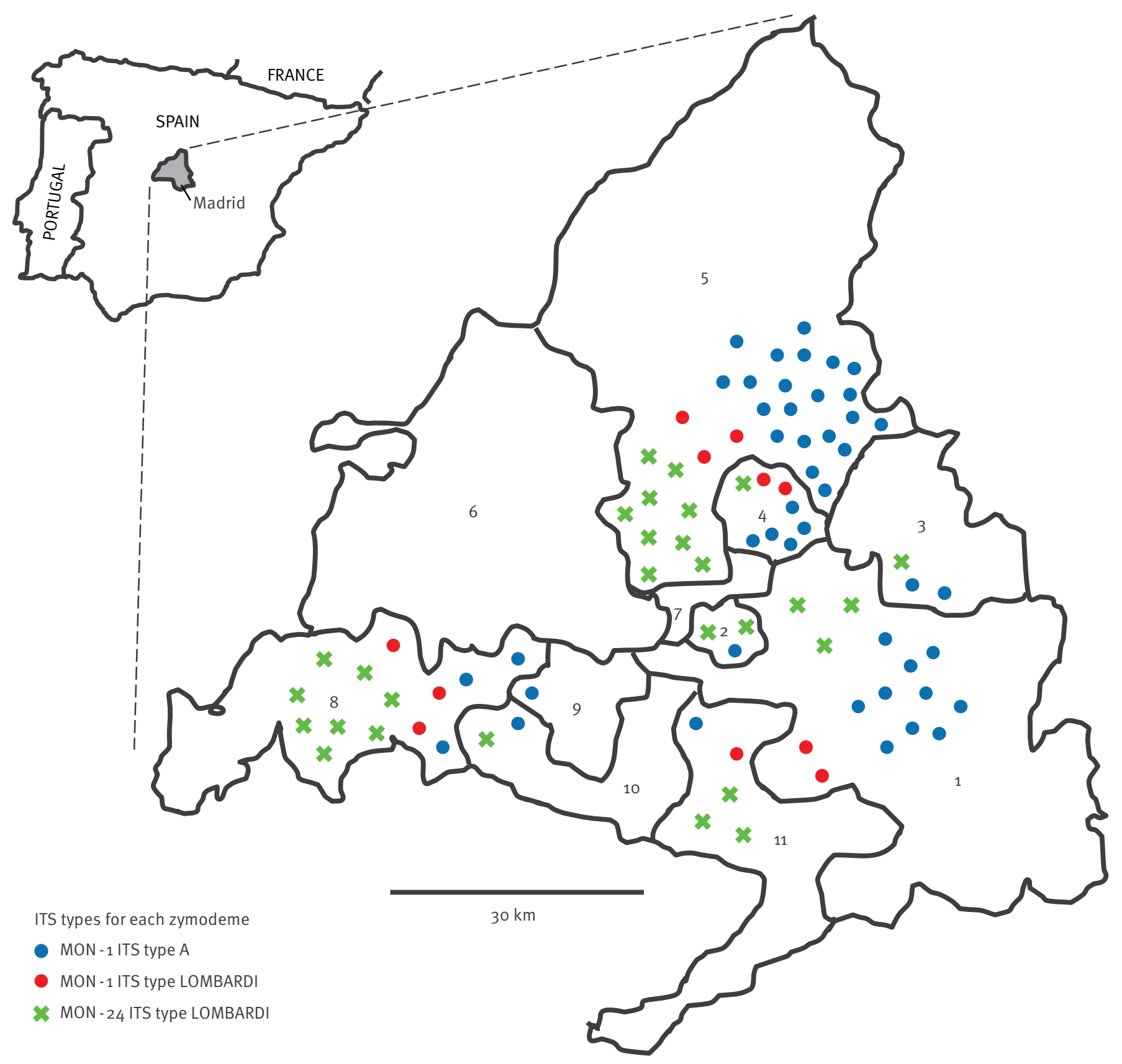

ITS: ribosomal internal transcribed spacers.

The numbers 1-11 represent the 11 Health Areas in Madrid. The ITS types for each zymodeme are shown. 


\section{FIGURE 3}

Partial alignment of the eight ITS sequence types for strains of the Leishmania donovani complex ${ }^{\mathrm{a}}$ and the ITS sequence type LOMBARDI identified in $52 \mathrm{~L}$. infantum isolates from Madrid, Spain, 2008-12

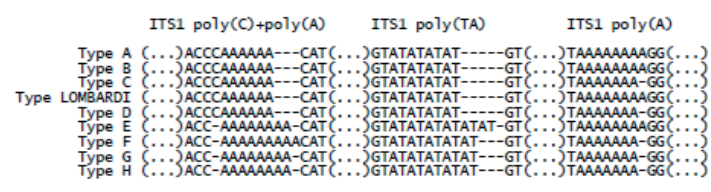

$24 \quad 39 \quad 61-76 \quad 124$

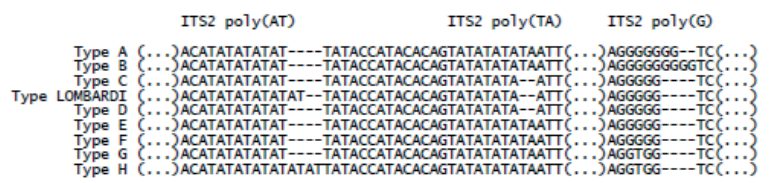

453

$495 \quad 740$

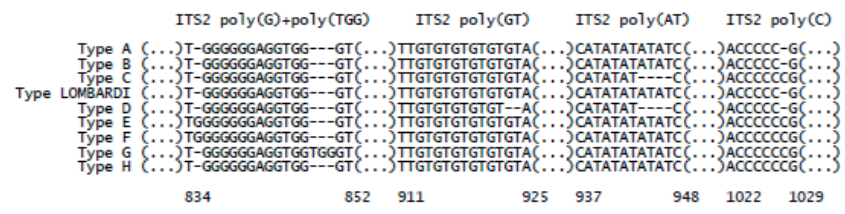

ITS: ribosomal internal transcribed spacers.

Differences between the sequence types are based solely on polymorphisms of the 12 microsatellites.

a The eight ITS types were described by Kuhls et al. [23]. Types $A$ and $B$ were described in $L$. infantum strains isolated in the Mediterranean basin, China and Brazil; type C in a L. donovani strain from China; types $D, E$ and $F$ in L. donovani strains from Ethiopia and Sudan; type G in L. donovani strains from Kenya and India and type $\mathrm{H}$ in $L$. donovani strains from India.

authors [23], ITS types A and B correspond to $L$. infantum from the Mediterranean basin, China and Brazil, ITS type $C$ corresponds to $L$. donovani from China, ITS types D, E and $F$ are associated with $L$. donovani from East Africa, ITS type $G$ is found in L. donovani from Kenya and India, and ITS type $\mathrm{H}$ in $L$. donovani from India.

\section{Results}

Analysis of the 12 microsatellite sites of the ITS 1 and ITS2 concatenated sequences returned two different ITS types among the 73 isolates in the first assembly (isolates from the leishmaniasis outbreak area and other regions of Madrid from 2008 to 2012). ITS type A was present in 21 of the isolates; the remaining 52 isolates were of a type that had not been described previously by Kuhls et al. [23]. The concatenated sequence of both ITS1 and ITS2 corresponding to this second ITS type was subjected to a BLASTn search [31], which returned the best score with a sequence corresponding to the $L$. infantum strain MHOM/ES/87/Lombardi (Gen Bank Accession Number AJoo0295). The 12 microsatellite sites found in MHOM/ES/87/Lombardi were identical to those observed in the second ITS type we found (hereafter called ITS-LOMBARDI). Comparison of ITS-LOMBARDI with the polymorphic microsatellite types described by Kuhls et al. for the $L$. donovani complex [23] is shown in Figure 3. ITS-LOMBARDI was common to all 31 isolates we analysed from the outbreak that affected Health Areas 9 and 10. These isolates were confirmed to be $L$. infantum by hsp7o PCR and DNA sequencing and further comparison to the DNA sequences of strains from the $L$. donovani complex (GenBank Accession Numbers FN395027-FN395033), described elsewhere [24].

For the second assembly ( $L$. infantum MON-1 and MON24 isolates from 1988 to 2005), 44 of the 55 L. infantum MON-1 isolates were ITS type A, while 11 were ITSLOMBARDI. All $28 \mathrm{~L}$. infantum MON-24 isolates were ITS-LOMBARDI.

In both assemblies, ITS-A and ITS-LOMBARDI sequence types were found in isolates from CL and VL cases, as well as in patients who were HIV-positive and -negative. Both ITS types are widespread in Madrid, having being detected in almost all Health Areas, since 1988 (ITS-A) and since 1992 (ITS-LOMBARDI) (Tables 2 and 3). ITS-LOMBARDI was found in all the L. infantum MON-24 isolates studied, but is not specific to this zymodeme, given that 11 of the $55 \mathrm{~L}$. infantum MON-1 isolates studied also had this ITS type.

Our phylogenetic analysis included ITS-LOMBARDI in a cluster - supported by a bootstrap value of $87 \%$ - with ITS types A, B, C and D. However, it is further separated from types $A$ and $B$ in a subsequent subcluster (Figure 4).

Capillary electrophoresis revealed seven different k26 PCR products sizes for the 73 isolates of the first assembly studied by this method, ranging from 584 to 962 base pairs (bp). A single PCR product was obtained for 65 of the 73 isolates, while the other eight showed a double PCR product of $920 \mathrm{bp}$ and $962 \mathrm{bp}$. When $k 26$ and ITS data were combined, again, seven genotypes were obtained, because ITS-A appears to be associated only with $k 26$ PCR products of $584-794 \mathrm{bp}$ and ITS-LOMBARDI with larger k26 PCR products (836-962 bp). Four combined genotypes were identified among the 32 isolates analysed from Health Areas 9 and 10 (31 of which were related to the outbreak), with L-920 (ITS-LOMBARDI in combination with a k26 PCR product of $920 \mathrm{bp}$ ) the most frequent (present in 23 of the 32 isolates). In the other Health Areas (those not affected by the outbreak), the most frequent combined genotype was A-626 (present in 17/41 isolates), followed by L-920 (present in 12/41 isolates).

No particular association was found between the combined genotypes and the HIV status of the patients or clinical form of leishamaniasis (cutaneous or visceral). Of the three cases with localised lymphadenopathy (LL), two were caused by the combined genotype L-920 


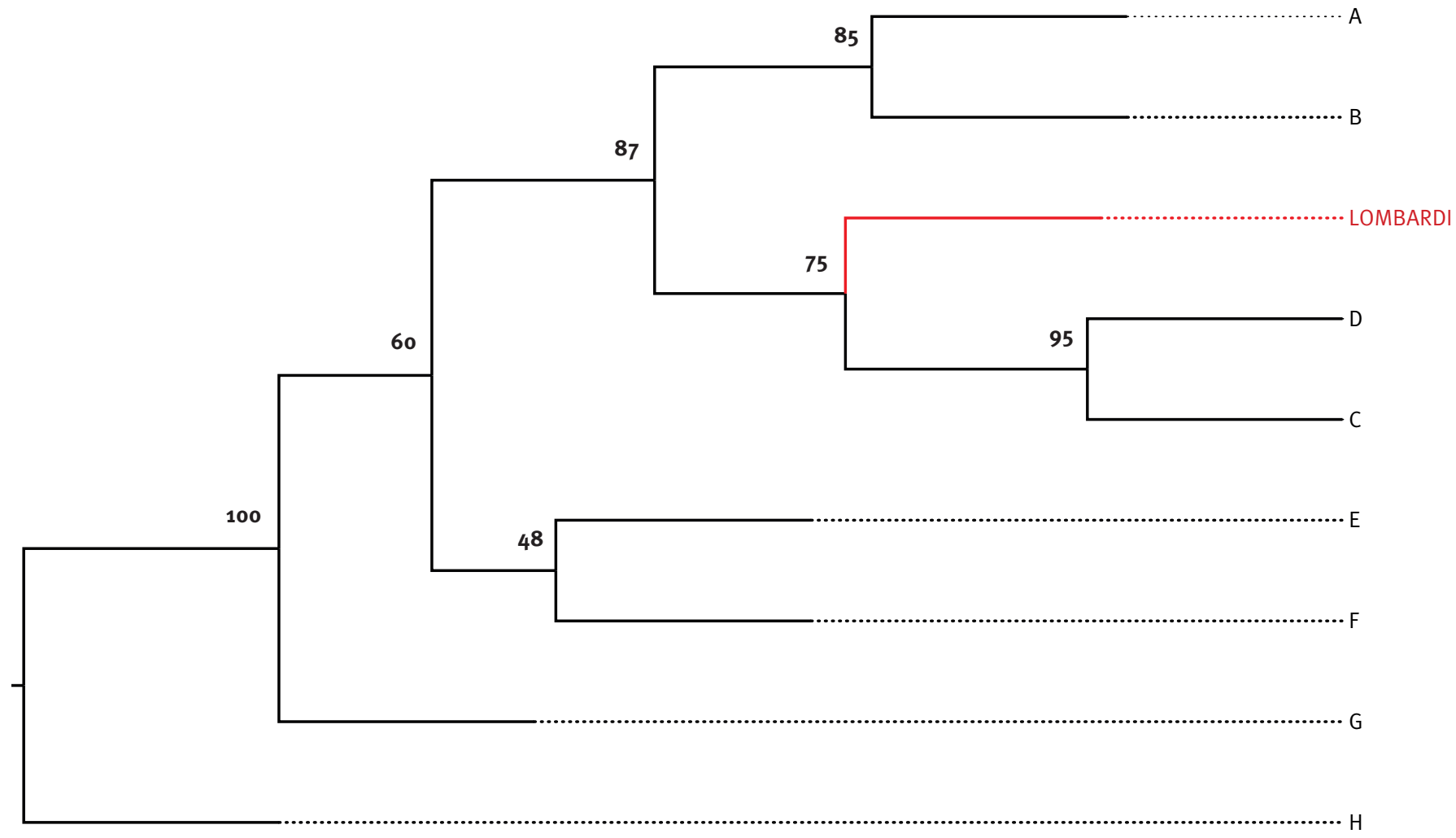

ITS: ribosomal internal transcribed spacers.

The most parsimonious tree found by heuristic search is presented. It was inferred by parsimony analysis of the nucleotide sequences of ITS1 and ITS2. The numbers above the branches indicate the percentages with which a given branch is supported in 10,000 bootstrap replications. ITS types A-H are those described by Kuhls et al. [23].

and one by L-962. The case with mucosal leishmaniasis (ML) was caused by L-962/920. Two of the LL cases and the ML case occurred in Health Area 9 (Table 2).

\section{Discussion}

To the best of our knowledge, this is the first molecular typing study of the leishmaniasis outbreak in Madrid that began in July 2009 and the most comprehensive molecular typing study carried out in Spain with isolates obtained within such a short period of time (2008-12) and small geographical area (Table 2). We also provided further information on $83 \mathrm{~L}$. infantum MON-1 and MON-24 isolates collected in Madrid during 1988 to 2005 .

A previous molecular typing study, which included L. infantum isolates from Madrid and other regions in Spain (isolated between 1986 and 1993), reported only the presence of ITS types A and B for this Leishmania species [23]. However, in our study $71 \%(52 / 73)$ of the $L$. infantum isolates from the first assembly had an ITS type, ITS-LOMBARDI, which had not been previously described. This ITS type was also present in all of the
MON-24 and $20 \%(11 / 55)$ of the MON-1 L. infantum isolates from the second assembly. It is noteworthy that this ITS type was also present in L. infantum MON-24 strain MHOM/ES/87/Lombardi, which was isolated in Spain (location unknown) in 1987, and its ITS DNA sequence (AJo00295) was submitted to the GenBank in year 1998, although no report on this ITS type was published. Unfortunately, neither this ITS sequence nor any other obtained from a MON-24 isolate were included by Kuhls et al. in their study of ITS sequence analysis of the L. donovani complex [23]. Our phylogenetic analysis, following a similar procedure, revealed that isolates with ITS-LOMBARDI, in spite of being L. infantum (as shown by MLEE and hsp7o gene DNA sequence analyses), do not form a clear phylogenetic group with ITS types A, B (Mediterranean basin, Brazil, China) and C (China), and are not well separated from the second main group that includes all strains from East Africa and India. ITS-LOMBARDI has been circulating in Spain (region unknown) since at least 1987 , based on the WHO code of the strain Lombardi (MHOM/ $\mathrm{ES} / 87 /$ Lombardi). According to our data on the first and second assemblies presented here, ITS-LOMBARDI is 
frequent in L. infantum isolates from Madrid and has been present in this region since at least 1992 (Table 3). L. infantum isolates obtained through xenodiagnosis from hares captured in an urban park during a study related to the Madrid outbreak described here were also typed as ITS-LOMBARDI [5] and this ITS type is also the only ITS type seen in isolates from all human cases associated with this outbreak that have been typed to date.

Another molecular typing study targeting the haspb (k26) gene included Spanish L. infantum isolates and revealed PCR product sizes of approximately $626 \mathrm{bp}$, $870 / 980 \mathrm{bp}, 870 / 1200 \mathrm{bp}$ and $870 / 1300 \mathrm{bp}$ [24]. In our study k26 PCR products sizes of 584-962 bp were found, with those of 626 and 920 bp being the most frequent, although among the outbreak-associated cases only k26 PCR products of 836-962 bp were found (Table 2). A product size of 920 bp was the most frequent.

The epidemiological picture of the leishmaniasis outbreak affecting Health Areas 9 and 10 is consistent with focal transmission of the parasite [16]. This, together with the fact that the $L$. infantum isolates obtained from patients in these areas presented mostly the combined genotype L-920, might suggest that an emerging, less common or 'new' L. infantum strain was the causative agent of the outbreak. Care must be taken, however, before drawing this conclusion. Molecular typing-based surveillance studies of leishmaniasis are scarce in Madrid and in other parts of Spain, and probably elsewhere too. Genotype L-920 has been isolated in another five of the 11 Health Areas of Madrid and has been present in two of them (Health Areas 5 and 8) since at least 2008. Additionally, ITS-LOMBARDI has been described in an isolate from Spain obtained in 1987, and in L. infantum MON-1 and MON-24 isolates from Madrid since 1992 and 1995, respectively, in different Health Areas (Table 3, Figure 2). We believe that more comprehensive molecular typing-based surveillance studies should be carried out in Spain, as well as in other leishmaniasis endemic countries. Given that leishmaniasis is currently re-emerging and spreading to previously non-endemic areas [21], activities aimed at bridging research with surveillance, as suggested by Dujardin et al. [9], will contribute to a better understanding of the epidemiology of leishmaniasis and will allow control strategies to be developed.

In terms of molecular typing, further studies are needed to ascertain the magnitude and origin of the outbreak in Madrid, particularly those aiming to identify the parasite genotypes circulating among sandflies, dogs and other alternative reservoirs: the WHO Collaborating Centre for Leishmaniasis is currently working on this. The simple analysis of the k26 PCR product size indicates that the outbreak was not caused by a single parasite strain (four combined genotypes were found). It seems more likely that the spread of a long-established transmission cycle from a nearby area, with its own degree of parasite diversity, into an area with a population with little or no immunity against Leishmania could have originated this outbreak.

In spite of the wide application of molecular methods to assess Leishmania population structure and to help in epidemiological studies [32], there are few opportunities to validate molecular markers for leishmaniasis outbreak investigations. We believe that the results here presented will contribute to this and, together with the material collected, we would be able to validate other approaches such as multilocus sequence typing or multilocus microsatellite typing, taking advantage of this 'experiment in nature' - the outbreak in south-west Madrid.

\section{Acknowledgements}

We would like to give thanks to our colleagues from the Subdirección General de Sanidad Ambiental y Epidemiología, Dirección General de Atención Primaria y Servicio de Epidemiología, and Servicio de Salud Pública del Área 9, Comunidad de Madrid (Spain), for their enthusiastic and collective nature during our collaboration on the studies of this outbreak. We would also like to thank our colleagues from referral hospitals which routinely contribute to our $L$. infantum cryo-bank. Last but not least we want to express our sincere gratitude and appreciation to the WHO Department for the Control of Neglected Tropical Diseases (HTM/NTD/IDM), Leishmaniasis Control Program, for its continuous support to the WHO Collaborating Centre for Leishmaniasis at the Instituto de Salud Carlos III. This study was supported in part by Instituto de Salud Carlos III (Grant number MPY-1248/12). Ivonne Pamela Llanes Acevedo is granted by Fundación para el Futuro de Colombia, COLFUTURO). 


\section{References}

1. Alvar J, Vélez ID, Bern C, Herrero M, Desjeux P, Cano J, et al. Leishmaniasis worldwide and global estimates of its incidence. PLoS One. 2012;7(5):e35671. http://dx.doi.org/10.1371/journal. pone.0035671 PMid:22693548 PMCid:PMC3365071

2. Chitimia L, Mu-oz-García Cl, Sánchez-Velasco D, Lizana V, Del Río L, Murcia L, et al. Cryptic Leishmaniosis by Leishmania infantum, a feature of canines only? A study of natural infection in wild rabbits, humans and dogs in southeastern Spain. Vet Parasitol. 2011;181(1):12-6. http://dx.doi.org/10.1016/j. vetpar.2011.04.016 PMid:21592669

3. Ramírez GA, Pe-afiel-Verdú C, Altimira J, García-González $B$, Vilafranca M. Naturally acquired visceral leishmaniosis in a captive Bennett's wallaby (Macropus rufogriseus rufogriseus). Vet Pathol. 2013;50(1):188-90. http://dx.doi. org/10.1177/0300985812446155 PMid:22692623

4. Sobrino R, Ferroglio E, Oleaga A, Romano A, Millan J, Revilla $M$, et al. Characterization of widespread canine leishmaniasis among wild carnivores from Spain. Vet Parasitol. 2008;155(34):198-203. http://dx.doi.org/10.1016/j.vetpar.2008.05.009 http://dx.doi.org/10.1016/j.vetpar.2008.05.003 PMid:18579311

5. Molina R, Jiménez MI, Cruz I, Iriso A, Martín-Martín I, Sevillano 0, et al. The hare (Lepus granatensis) as potential sylvatic reservoir of Leishmania infantum in Spain. Vet Parasitol. 2012;19o(1-2):268-71. http://dx.doi.org/10.1016/j. vetpar.2012.05.006 PMid:22677135

6. de la Loma A, Alvar J, Martinez Galiano E, Blázquez J, Alcalá Mu-oz A, Nájera R. Leishmaniasis or AIDS? Trans R Soc Trop Med Hyg. 1985;79(3):421-2. http://dx.doi. org/10.1016/0035-9203(85)90400-6

7. Desjeux P, Alvar J. Leishmania/HIV co-infections: epidemiology in Europe. Ann Trop Med Parasitol. 2003;97 Suppl 1:3-15. http://dx.doi.org/10.1179/000349803225002499 PMid:14678629

8. Alvar J, Aparicio P, Aseffa A, Den Boer M, Ca-avate C, Dedet JP, et al. The relationship between leishmaniasis and AIDS: the second 10 years. Clin Microbiol Rev. 2008;21(2):334-59. http://dx.doi.org/10.1128/CMR.00061-07 PMid:18400800 PMCid:PMC2292576

9. Dujardin JC, Campino L, Ca-avate C, Dedet JP, Gradoni L, Soteriadou K, et al. Spread of vector-borne diseases and neglect of Leishmaniasis, Europe. Emerg Infect Dis. 2008;14(7):1013-8. http://dx.doi.org/10.3201/eid1407.071589 PMid:18598618 PMCid:PMC2600355

10. Ready PD. Leishmaniasis emergence in Europe. Euro Surveill. 2010;15(10):pii=19505. Available from: http://www. eurosurveillance.org/ViewArticle. aspx?Articleld $=19505$ PMid:20403308

11. Maroli M, Rossi L, Baldelli R, Capelli G, Ferroglio E, Genchi $C$, et al. The northward spread of leishmaniasis in Italy: evidence from retrospective and ongoing studies on the canine reservoir and phlebotomine vectors. Trop Med Int Health. 2008;13(2):256-64.

http://dx.doi.org/10.1111/j.1365-3156.2007.01998.x PMid:18304273

12. Biglino A, Bolla C, Concialdi E, Trisciuoglio A, Romano A, Ferroglio E. Asymptomatic Leishmania infantum infection in an area of northwestern Italy (Piedmont region) where such infections are traditionally nonendemic. J Clin Microbiol. 2010;48(1):131-6.

http://dx.doi.org/10.1128/JCM.00416-09 PMid:19923480 PMCid:PMC2812267

13. Miró G, Checa R, Montoya A, Hernández L, Dado D, Gálvez $R$. Current situation of Leishmania infantum infection in shelter dogs in northern Spain. Parasit Vectors. 2012;5:60. http://dx.doi.org/10.1186/1756-3305-5-60 PMid:22452948 PMCid:PMC3348073

14. Farkas R, Tánczos B, Bongiorno G, Maroli M, Dereure Ready PD. First surveys to investigate the presence of canine leishmaniasis and its phlebotomine vectors in Hungary. Vector Borne Zoonotic Dis. 2011;11(7):823-34. http://dx.doi.org/10.1089/vbz.2010.0186 PMid:21254904

15. Tánczos B, Balogh N, Király L, Biksi I, Szeredi L, Gyurkovsky $M$, et al. First record of autochthonous canine leishmaniasis in Hungary. Vector Borne Zoonotic Dis. 2012;12(7):588-94 http://dx.doi.org/10.1089/vbz.2011.0906 PMid:22607079 PMCid:PMC3398396

16. Servicio de Epidemiología de la Comunidad de Madrid. Brote comunitario de leishmaniasis en la zona suroeste de la Comunidad de Madrid, 2009-2012. [Community outbreak of leishmaniasis in the southwest region of Madrid Autonomous Community]. Boletín Epidemiológico de la Comunidad de Madrid. 2011;17(12):25-32. Spanish. Available from: http:// www.madrid.org/cs/Satellite?blobcol=urldata\&blobheader $=a$ pplication\%2Fpdf\&blobheadername1=Content-Disposition\&bl obheadervalue $1=$ filename\%3DDiciembre2011. pdf\&blobkey=id \&blobtable $=$ MungoBlobs $\&$ blobwhere $=1310962031549 \&$ ssbin ary $=$ true

17. Arce A, Estirado A, Ordobas M, Sevilla S, García N, Moratilla L, et al. Re-emergence of leishmaniasis in Spain: community outbreak in Madrid, Spain, 2009 to 2012. Euro Surveill. 2013;18(30):pii=20546. Available from: http://www. eurosurveillance.org/ViewArticle.aspx?Articleld $=20546$

18. Chicharro C, Jiménez MI, Alvar J. Iso-enzymatic variability of Leishmania infantum in Spain. Ann Trop Med Parasitol. 2003;97 Suppl 1:57-64. http://dx.doi. org/10.1179/000349803225002534 PMid:14678633

19. Cruz I, Chicharro C, Nieto J, Bailo B, Ca-avate C, Figueras MC, et al. Comparison of new diagnostic tools for management of pediatric Mediterranean visceral leishmaniasis. J Clin Microbiol. 2006;44(7):2343-7. http://dx.doi.org/10.1128/ JCM.02297-05 PMid:16825347 PMCid:PMC1489479

20. Pratlong F, Rioux JA, Marty P, Faraut-Gambarelli F, Dereure J, Lanotte G, et al. Isoenzymatic analysis of 712 strains of Leishmania infantum in the south of France and relationship of enzymatic polymorphism to clinical and epidemiological features. J Clin Microbiol. 2004;42(9):4077-82. http://dx.doi. org/10.1128/JCM.42.9.4077-4082.2004 PMid:15364993 PMCid:PMC516332

21. World Health Organization (WHO). Control of the leishmaniases: report of a meeting of the WHO Expert Committee on the Control of Leishmaniases, Geneva, 22-26 March 2010. Geneva: WHO; 2010. WHO technical report series; no. 949. Available from: http://whqlibdoc.who.int/trs/WHO_ TRS 949_eng.pdf

22. Schönian G, Kuhls K, Mauricio IL. Molecular approaches for a better understanding of the epidemiology and population genetics of Leishmania. Parasitology. 2011;138(4):405-25. http://dx.doi.org/10.1017/So031182010001538 PMid:21078222

23. Kuhls K, Mauricio IL, Pratlong F, Presber W, Schönian G. Analysis of ribosomal DNA internal transcribed spacer sequences of the Leishmania donovani complex. Microbes Infect. 2005;7(11-12):1224-34. http://dx.doi.org/10.1016/j. micinf.2005.04.009 PMid:16002315

24. Haralambous C, Antoniou M, Pratlong F, Dedet JP, Soteriadou K. Development of a molecular assay specific for the Leishmania donovani complex that discriminates L. donovani/ Leishmania infantum zymodemes: a useful tool for typing MON-1. Diagn Microbiol Infect Dis. 2008;60(1):33-42. http:// dx.doi.org/10.1016/j.diagmicrobio.2007.07.019 PMid:17889482

25. Fraga J, Montalvo AM, De Doncker S, Dujardin JC, Van der Auwera G. Phylogeny of Leishmania species based on the heatshock protein 70 gene. Infect Genet Evol. 2010;10(2):238-45. http://dx.doi.org/10.1016/j.meegid.2009.11.007 PMid:19913110

26. Hall TA. BioEdit: a user-friendly biological sequence alignment editor and analysis program for Windows 95/98/NT. Nucleic Acids Symp Ser. 1999;41:95-8.

27. Depledge DP, Maclean LM, Hodgkinson MR, Smith BA, Jackson AP, Ma S, et al. Leishmania-specific surface antigens show sub-genus sequence variation and immune recognition. PLoS Negl Trop Dis. 2010;4(9):e829. http://dx.doi.org/10.1371/ journal.pntd.0000829 PMid:20927190 PMCid:PMC2946902

28. Zackay A, Nasereddin A, Takele Y, Tadesse D, Hailu W, Hurissa Z, et al. Polymorphism in the HASPB repeat region of East African Leishmania donovani strains. PLoS Negl Trop Dis. 2013;7(1):e2031. http://dx.doi.org/10.1371/journal. pntd.0002031 PMid:23358849 PMCid:PMC3554577

29. Bhattacharyya T, Boelaert M, Miles MA. Comparison of visceral leishmaniasis diagnostic antigens in African and Asian Leishmania donovani reveals extensive diversity and regionspecific polymorphisms. PLos Negl Trop Dis. 2013;7(2):e2057. http://dx.doi.org/10.1371/journal.pntd.0002057 PMid:23469296 PMCid:PMC3585016

30. Felsenstein J. PHYLIP-Phylogeny Inference Package (version 3.2). Cladistics.1989; 5: 164-6.

31. BLAST. Basic local alignment search tool. [Accessed 15 Mar 2012]. Available from: http://blast.ncbi.nlm.nih.gov/

32. Schönian G, Mauricio I, Gramiccia M, Ca-avate C, Boelaert M, Dujardin JC. Leishmaniases in the Mediterranean in the era of molecular epidemiology. Trends Parasitol. 2008;24(3):135-42. http://dx.doi.org/10.1016/j.pt.2007.12.006 PMid:18262469 